\title{
Design of a triple-bend isochronous achromat with minimum coherent-synchrotron-radiation-induced emittance growth
}

\author{
M. Venturini \\ Lawrence Berkeley National Laboratory, University of California, Berkeley, California 94720, USA
}

(Received 8 April 2015; revised manuscript received 15 April 2016; published 9 June 2016)

\begin{abstract}
Using a 1D steady-state free-space coherent synchrotron radiation (CSR) model, we identify a special design setting for a triple-bend isochronous achromat that yields vanishing emittance growth from CSR. When a more refined CSR model with transient effects is included in the analysis, numerical simulations show that the main effect of the transients is to shift the emittance growth minimum slightly, with the minimum changing only modestly.
\end{abstract}

DOI: 10.1103/PhysRevAccelBeams.19.064401

\section{INTRODUCTION}

It is desirable for acceleration and transport of highbrightness electron bunches to occur without degradation of the beam quality. Unfortunately, a number of processes can spoil the beam transverse emittance and among these one of the most prevalent is coherent synchrotron radiation (CSR). As the electrons in a bunch travel through a bend, synchrotron radiation at the low end of the frequency spectrum is emitted coherently, perturbing the particle energy, inducing transverse offsets both in the spacial and angular coordinates, and therefore causing projected emittance growth.

One way to contain the adverse effects of CSR is to reduce overall bending; however, to eliminate bending altogether is usually not an option. For instance, in single-pass systems for free electron lasers (FELs) dipole magnets are required for bunch compression and often to distribute the electrons to off-axis beamlines. In multipass systems, such as energy recovery linacs, bending is integral to the machine topology.

Here we consider the problem of minimizing CSR effects on the transverse emittance in a triple-bend isochronous achromat, a lattice unit widely used in accelerator design. We adopt a 1D steady-state free-space model of CSR [1] and a method of analysis first introduced in [2] for the study of CSR in bunch compressors. We show that within the approximation of the model it is possible to specify a lattice design that yields vanishing CSR-induced emittance growth.

Our approach has some similarities with $[3,4]$ and in particular [5]. We refer to the Introduction in [5] for a review of various approaches to the problem of minimizing CSR-effects on the emittance. For additional related work see also [6-12].

Published by the American Physical Society under the terms of the Creative Commons Attribution 3.0 License. Further distribution of this work must maintain attribution to the author(s) and the published article's title, journal citation, and DOI.

\section{FORMALISM}

Consider a dispersive beam line from $s=s_{i}$ to $s=s_{f}$ with bending occurring in the horizontal plane and no acceleration. In a 1D approximation, the effect of CSR on a particle of the bunch at location $s$ along the beam line is to induce a relative-energy change $\delta_{s}(z)$ depending on the arclength coordinate $s$ and particle longitudinal coordinate $z$.

In the linear approximation, the particle orbit in the horizontal plane following the energy kick evolves according to

$$
\begin{gathered}
x=R_{11}^{s_{i} \rightarrow s_{f}} x_{i}+R_{12}^{s_{i} \rightarrow s_{f}} x_{i}^{\prime}+R_{16}^{s \rightarrow s_{f}} \delta_{s}(z), \\
x^{\prime}=R_{21}^{s_{i} \rightarrow s_{f}} x_{i}+R_{22}^{s_{i} \rightarrow s_{f}} x_{i}^{\prime}+R_{26}^{s \rightarrow s_{f}} \delta_{s}(z),
\end{gathered}
$$

where $R_{i j}$ are the entries of the linear transport matrix while $x_{i}, x_{i}{ }^{\prime}$ and $x, x^{\prime}$ are the particle coordinates at the entrance and exit of the beam line respectively. Notice that the entries $R_{11}, R_{12}, R_{21}$, and $R_{22}$ are for the transport matrix from $s_{i}$ to $s_{f}$, whereas the entries $R_{16}$ an $R_{26}$ are for transport starting from $s_{i} \leq s \leq s_{f}$, where the CSR energy kick occurs.

Integrating the effect of CSR through the whole dispersive section, the particle coordinates at the exit of the beam line become $x=x_{\beta}+\hat{x}(z)$ and $x^{\prime}=x_{\beta}{ }^{\prime}+\hat{x}^{\prime}(z)$ with $x_{\beta}=R_{11}^{s_{i} \rightarrow s_{f}} x_{i}+R_{12}^{s_{i} \rightarrow s_{f}} x_{i}^{\prime}$ and $x_{\beta}^{\prime}=R_{21}^{s_{i} \rightarrow s_{f}} x_{i}+R_{22}^{s_{i} \rightarrow s_{f}} x_{i}^{\prime}$, and

$$
\begin{gathered}
\hat{x}(z) \equiv \int_{s_{i}}^{s_{f}} \frac{d \delta_{s}(z)}{d s} R_{16}^{s \rightarrow s_{f}} d s, \\
\hat{x}^{\prime}(z) \equiv \int_{s_{i}}^{s_{f}} \frac{d \delta_{s}(z)}{d s} R_{26}^{s \rightarrow s_{f}} d s .
\end{gathered}
$$

If the beam is initially centered, $\left\langle x_{i}\right\rangle=\left\langle x_{i}{ }^{\prime}\right\rangle=0$, where $\langle\cdot\rangle$ represents averaging over the bunch population in phase-space, we can think of $\left(\hat{x}(z), \hat{x}^{\prime}(z)\right)$ as the centroid 
coordinates of the beam thin slice centered at $z$. If the initial beam has vanishing transverse emittance $\left(x_{\beta}=x_{\beta}{ }^{\prime}=0\right)$ at the exit of the beam line the beam is represented by a onedimensional curve in the horizontal phase space described parametrically by the $\left(\hat{x}(z), \hat{x}^{\prime}(z)\right)$ pair.

Assuming that the beam at $s_{i}$ is centered, we average over the full $6 \mathrm{D}$ phase-space at $s_{f}$ to obtain the 2ndmoment of the beam distribution in the horizonal spacial coordinate:

$$
\begin{aligned}
\left\langle(x-\langle x\rangle)^{2}\right\rangle & =\left\langle\left(x_{\beta}-\left\langle x_{\beta}\right\rangle+\hat{x}-\langle\hat{x}\rangle\right)^{2}\right\rangle \\
& =\left\langle\left(x_{\beta}+\Delta x\right)^{2}\right\rangle=\left\langle x_{\beta}^{2}\right\rangle+\left\langle(\Delta x)^{2}\right\rangle,
\end{aligned}
$$

having introduced the notation $\Delta x \equiv \hat{x}-\langle\hat{x}\rangle$, and exploited the lack of correlation (justified in a 1D model) between the CSR-induced energy kick and the transverse coordinate, $\left\langle x_{\beta} \Delta x\right\rangle=0$.

We have

$$
\begin{aligned}
\left\langle(\Delta x)^{2}\right\rangle= & \left\langle\hat{x}^{2}\right\rangle-\langle\hat{x}\rangle^{2} \\
= & \int d z \lambda(z)\left[\int \frac{d \delta_{s}(z)}{d s} R_{16}^{s \rightarrow s_{f}} d s\right]^{2} \\
& -\left[\int d z \lambda(z) \int \frac{d \delta_{s}(z)}{d s} R_{16}^{s \rightarrow s_{f}} d s\right]^{2},
\end{aligned}
$$

where $\lambda(z)$ is the beam longitudinal density normalized to unity. Similar expressions hold for $\left\langle\left(\Delta x^{\prime}\right)^{2}\right\rangle$ and $\left\langle\Delta x \Delta x^{\prime}\right\rangle$. From now on, to avoid notational clutter we will drop the parentheses and write $\left\langle\Delta x^{2}\right\rangle$ instead of $\left\langle(\Delta x)^{2}\right\rangle$, etc.

We now specialize these expressions to the case of interest here and assume that (i) the longitudinal density of the bunch is stationary or close to stationary, (ii) CSR is represented by a steady-state model neglecting transient effects through entrance and exit of the bends, and (iii) all bends (modeled in the hard-edge approximation) have the same nominal magnetic field. Under these assumptions the $z$ dependence is factored out and we have $\frac{d \delta_{s}(z)}{d s} \equiv$ $h_{B}(s) \hat{\delta}^{\prime}(z)$ where $h_{B}(s)=1$ for $s$ in a bend and $h_{B}(s)=0$ elsewhere, and

$$
\hat{\delta}^{\prime}(z)=\frac{2 r_{c} N_{b}}{3^{1 / 3} R^{2 / 3} \gamma} \int_{z}^{\infty} \frac{d \lambda\left(z^{\prime}\right)}{d z^{\prime}} \frac{d z^{\prime}}{\left(z^{\prime}-z\right)^{1 / 3}} .
$$

In the above expression $R$ is the dipole radius of curvature, $N_{b}$ the bunch population, $m$ the electron mass, $r_{c}$ the electron classical radius, and $\gamma$ the relativistic factor. Notice that we are adopting the convention that a particle in the bunch head has $z<0 ; \delta^{\prime}(z)>0$ denotes energy gain. The above expression (6) simplifies to

$$
\left\langle\Delta x^{2}\right\rangle=\left[\int_{B} R_{16}^{s \rightarrow s_{f}} d s\right]^{2}\left\langle\Delta \hat{\delta}^{\prime 2}\right\rangle
$$

where $\left\langle\Delta \hat{\delta}^{\prime 2}\right\rangle=\left\langle\hat{\delta}^{\prime 2}\right\rangle-\left\langle\hat{\delta}^{\prime}\right\rangle^{2} \equiv\left(d \sigma_{\delta} / d s\right)^{2}$ is the square of the rms relative-energy spread per unit arc length induced by CSR along a dipole. Similarly

$$
\begin{gathered}
\left\langle\Delta x^{\prime 2}\right\rangle=\left[\int_{B} R_{26}^{s \rightarrow s_{f}} d s\right]^{2}\left\langle\Delta \hat{\delta}^{\prime 2}\right\rangle, \\
\left\langle\Delta x \Delta \hat{x}^{\prime}\right\rangle=\left[\int_{B} R_{16}^{s \rightarrow s_{f}} d s\right]\left[\int_{B} R_{26}^{s \rightarrow s_{f}} d s\right]\left\langle\Delta \hat{\delta}^{\prime 2}\right\rangle .
\end{gathered}
$$

The subscript " $B$ " on the integral signs emphasizes that the integrals extend only over the bending magnets.

The rms projected (geometric) emittance is defined as the determinant of the covariance matrix

$\sigma=\left(\begin{array}{cc}\left\langle(x+\Delta x)^{2}\right\rangle & \left\langle(x+\Delta x)\left(x^{\prime}+\Delta x^{\prime}\right)\right\rangle \\ \left\langle(x+\Delta x)\left(x^{\prime}+\Delta x^{\prime}\right)\right\rangle & \left\langle\left(x^{\prime}+\Delta x^{\prime}\right)^{2}\right\rangle\end{array}\right)$.

Observed at the exit $s_{f}$ of the beam line the emittance reads

$$
\begin{aligned}
\varepsilon_{x}^{2}= & \varepsilon_{x 0}^{2}+\varepsilon_{x 0}\left(\beta_{x}\left\langle\Delta x^{\prime 2}\right\rangle\right. \\
& \left.+2 \alpha_{x}\left\langle\Delta x \Delta x^{\prime}\right\rangle+\gamma_{x}\left\langle\Delta x^{2}\right\rangle\right)+\Delta \varepsilon_{x}^{2}
\end{aligned}
$$

where $\varepsilon_{x 0}^{2}$ is the beam unperturbed emittance, $\alpha_{x}, \beta_{x}, \gamma_{x}$ are the Twiss functions at $s_{f}$, and

$$
\Delta \varepsilon_{x}^{2}=\left\langle\Delta x^{2}\right\rangle\left\langle\Delta x^{\prime 2}\right\rangle-\left\langle\Delta x \Delta x^{\prime}\right\rangle^{2} .
$$

Notice that from (8), (9), and (10), it follows that $\left\langle\Delta x \Delta x^{\prime}\right\rangle^{2}=\left\langle\Delta x^{2}\right\rangle\left\langle\Delta x^{\prime 2}\right\rangle$ and therefore $\Delta \varepsilon_{x}^{2}=0$. We should point out that this is not a general result: it strictly depends on the assumption that the longitudinal beam density remains stationary, in which case the set $(\hat{x}(z)$, $\left.\hat{x}^{\prime}(z)\right)$ describes a zero-area straight segment in the horizontal phase space (see Fig. 5). In contrast, downstream of a magnetic chicane compressor $\Delta \varepsilon_{x}^{2}$ can be significant.

We can reduce emittance growth by either choosing $\alpha_{x}$, $\beta_{x}, \gamma_{x}$ appropriately (see Appendix A) or minimizing the second moments $\left\langle\Delta \hat{x}^{2}\right\rangle,\left\langle\Delta \hat{x}^{\prime 2}\right\rangle$ and $\left\langle\Delta \hat{x} \Delta \hat{x}^{\prime}\right\rangle$. Equation (12) indicates no emittance growth if $\left\langle\Delta \hat{x}^{2}\right\rangle$ and $\left\langle\Delta \hat{x}^{\prime 2}\right\rangle$ can be set to zero simultaneously, i.e.,

$$
\begin{aligned}
& \int_{B} R_{16}^{s \rightarrow s_{f}} d s=0 \\
& \int_{B} R_{26}^{s \rightarrow s_{f}} d s=0 .
\end{aligned}
$$

As shown in the next section, in a three-bend isochronous achromat these integrals can indeed be made vanish simultaneously through second order in the bend angle. 


\section{APPLICATION TO 3-BEND ISOCHRONOUS ACHROMATS}

Our midpoint symmetric beam line consists of three identical bending magnets equally spaced, see schematic in Fig. 1. We adopt a small bend-angle approximation for the transfer matrix of the three dipoles obtained from the analytical expressions in the hard-edge approximation [13] by Taylor expanding with respect to the dipole bending angle $\theta$ (while keeping $L_{B}=R / \theta$ constant). To lowest order and in the ultrarelativistic approximation the transfer matrix for motion in the horizontal and longitudinal planes reads

$$
R_{B}=\left(\begin{array}{cccc}
1 & L_{B} & 0 & L_{B} \theta / 2 \\
0 & 1 & 0 & \theta \\
\theta & L_{B} \theta / 2 & 1 & L_{B} \theta^{2} / 6 \\
0 & 0 & 0 & 1
\end{array}\right)
$$

To this order $R_{B}$ can be thought of as describing the action of either a rectangular or sector-bend magnet. Incidentally, notice that the entry for the momentum compaction is positive $\left(R_{B}\right)_{16}=L_{B} \theta^{2} / 6>0$, consistent with the convention that a particle in the bunch head has $z<0$.

Of interest is also the transfer matrix through half of the dipole $R_{B / 2}$, obtained from (16) by replacing $L_{B} \rightarrow L_{B} / 2$ and $\theta \rightarrow \theta / 2$.

In the most general terms we write the transfer matrix $M$ from the exit of the first dipole to the entrance of the second as

$$
M=\left(\begin{array}{cccc}
m_{11} & m_{12} & 0 & 0 \\
m_{21} & m_{22} & 0 & 0 \\
0 & 0 & 1 & 0 \\
0 & 0 & 0 & 1
\end{array}\right)
$$

The transfer map for the whole achromat line can then be written as $R^{s_{i} \rightarrow s_{f}}=A^{I I} A^{I}$ where $A^{I}$ and $A^{I I}$ are the transfer

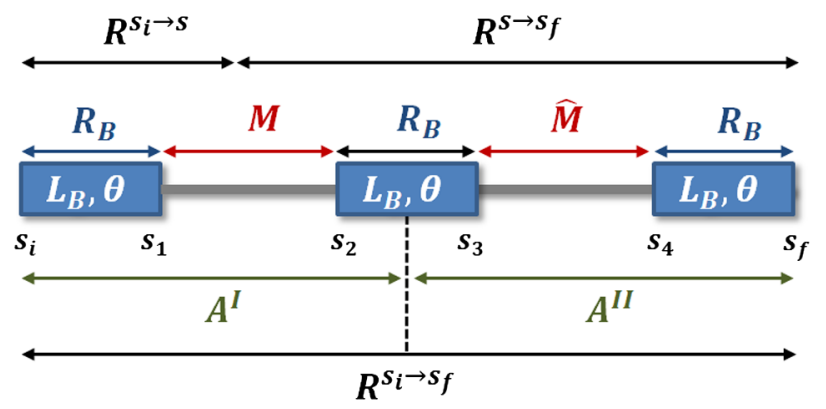

FIG. 1. Schematic of a 3-bend achromat with midpoint symmetry consisting of three identical dipoles with $L_{B}$ length and $\theta$ bending angle. Indicated is our notation for the transfer matrices through specified beam line sections. The quadrupoles providing the necessary focusing between the dipoles are not shown. matrices through the first and second half of the beam line. We have in particular

$$
A^{I}=R_{B / 2} M R_{B}
$$

and similarly $A^{I I}=R_{B} \hat{M} R_{B / 2}$, where $\hat{M}$ has the same entries as $M$, but with $m_{11}$ and $m_{22}$ exchanged.

The four entries of the $M$ matrix are the variables available for minimizing the emittance growth, whereas the dipole magnet length $L_{B}$ and bending angle $\theta$ are set by the lattice designer by considerations unrelated to CSR. One degree of freedom is taken by imposition of the achromatic condition $A_{26}^{I}=0$ (i.e. vanishing derivative of the dispersion function at the midpoint. Because the dispersion function and its derivative are zero at the entrance of the beam line, midpoint symmetry and $A_{26}^{I}=0$ imply that they will vanish at the exit of the beam line as well). A second degree of freedom is needed to enforce isochronicity $\left(A_{56}^{I}=0\right)$, and a third is taken by the symplectic condition. Remarkably, the one degree of freedom left is sufficient to satisfy both Eqs. (14) and (15).

In more detail, through second order in $\theta$ the achromatic, isochronous, and symplectic conditions imposed upon Eq. (18) translate into

$$
\begin{gathered}
m_{12}=L_{B}\left(3-m_{21} L_{B}\right) / 4 \\
m_{11}=-\left(L_{B}+4 m_{12}\right) / 2 L_{B} \\
m_{22}=-\left(1+m_{21} L_{B}\right) / 2
\end{gathered}
$$

leaving one free parameter, e.g., $m_{21}$. The next step is to work out the expressions for $R_{16}^{s \rightarrow s_{f}}$ and $R_{26}^{s \rightarrow s_{f}}$ and their integrals over the three bending magnets, see Appendix B. The final result for the integrals, valid through second order in $\theta$, is

$$
\begin{array}{r}
\int_{B} R_{16}^{s \rightarrow s_{f}} d s=-\frac{L_{B}^{2} \theta}{24}\left(15-2 m_{21} L_{B}\right)\left(1-m_{21} L_{B}\right), \\
\int_{B} R_{26}^{s \rightarrow s_{f}} d s=\frac{m_{21} L_{B}^{2} \theta}{12}\left(15-2 m_{21} L_{B}\right) .
\end{array}
$$

We observe that choosing $m_{21}=15 / 2 L_{B}$ makes both (22) and (23) vanish. We should emphasize that in this case the model predicts no emittance growth regardless of the values set for the Twiss functions at the beam line ends.

The result can be generalized to triple bend achromats with midpoint symmetry but with the middle dipole having different length and bend angle from those of the other two dipoles. This relaxes the assumption that the radius of curvature be identical for all dipoles. The expression for $m_{21}$ that makes the two above integral vanish is 


$$
m_{21}=\frac{3 t\left(2+\ell^{1 / 3} t^{2 / 3}\right)\left(4+\ell t^{2}\right)}{2\left(2+\ell t^{2}\right) L_{B}}
$$

where $\ell L_{B}$ and $t \theta$ are the middle dipole length and bend angle $\left(L_{B}\right.$ and $\theta$ being length and bend angle of the two outer dipoles).

The relevant entries of the transfer matrix for the whole achromat are then

$$
\begin{gathered}
R_{11}^{s_{i} \rightarrow s_{f}}=\left[3 t^{2}+m_{21}^{2} L_{B}^{2}\left(2+\ell t^{2}\right)-m_{21} L_{B} t\left(8+\ell t^{2}\right)\right] / 3 t^{2}, \\
R_{21}^{s_{i} \rightarrow s_{f}}=2 m_{21}\left[\left(2+\ell t^{2}\right) m_{21} L_{B}-6\right] / 3 t^{2} \\
R_{22}^{s_{i} \rightarrow s_{f}}=R_{11}^{s_{i} \rightarrow s_{f}},
\end{gathered}
$$

and $R_{12}=\left(R_{11} R_{22}-1\right) / R_{21}$ can be derived by the symplectic condition.

\section{NUMERICAL EXAMPLE}

For illustration we discuss a numerical example loosely inspired by the lattice in the spreader design for the Next Generation Light Source [14], which motivated this study, see Table I.

We consider a beam with Gaussian density profile $\lambda(z)$, rms length $\sigma_{z}$, and specialize the calculation to the case of symmetric beta function through the beam line: $\beta_{x}\left(s_{f}\right)=$ $\beta_{x}\left(s_{i}\right)$ and $\alpha_{x}\left(x_{f}\right)=-\alpha_{x}\left(x_{i}\right)$.

To this end, we first write the transfer matrix through the whole beam line $R^{s_{i} \rightarrow s_{f}}$ :

$$
\begin{gathered}
R_{11}^{s_{i} \rightarrow s_{f}}=m_{21}^{2} L_{B}^{2}-3 m_{21} L_{B}+1, \\
R_{12}^{s_{i} \rightarrow s_{f}}=\left(m_{21}^{2} L_{B}^{3}-4 m_{21} L_{B}^{2}+3 L_{B}\right) / 2, \\
R_{21}^{s_{i} \rightarrow s_{f}}=2 m_{21}^{2} L_{B}-4 m_{21}, \\
R_{22}^{s_{i} \rightarrow s_{f}}=R_{11}^{s_{i} \rightarrow s_{f}} .
\end{gathered}
$$

Using the general parametrization for symplectic transport matrices in terms of Twiss functions [13] at the two beam line ends [see also Eq. (A3) in Appendix A], we write $\beta_{x}\left(s_{f}\right)=R_{12}^{s_{i} \rightarrow s_{f}} / \sin \psi$ and $\alpha_{x}\left(s_{f}\right)=-\left[R_{11}^{s_{i} \rightarrow s_{f}}-\cos \psi\right] /$ $\sin \psi$. Having the freedom to set the phase we write

TABLE I. Selected parameters for the ELEGANT simulation.

\begin{tabular}{lc}
\hline \hline Dipole length, $L_{B}$ & $0.9 \mathrm{~m}$ \\
Bending angle, $\theta$ & $0.176 \mathrm{rad}$ \\
Beam energy & $2.4 \mathrm{GeV}$ \\
Unperturbed norm. emittance, $\gamma \varepsilon_{x 0}$ & $0.6 \mu \mathrm{m}$ \\
Bunch charge & $300 \mathrm{pC}$ \\
Bunch length, $\sigma_{z}$ & $0.04 \mathrm{~mm}$ \\
\hline \hline
\end{tabular}

$\psi=\pi / 2$, which minimizes $\left|\alpha_{x}\left(s_{f}\right)\right|$, yielding $\beta_{x}\left(s_{f}\right)=$ $L_{b}\left(3-4 L_{b} m_{21}+L_{b}^{2} m_{21}^{2}\right) / 2$, and $\alpha_{x}\left(s_{f}\right)=-L_{B}^{2} m_{21}^{2}+$ $3 L_{B} m_{21}-1$.

We can now write an analytical expression for the emittance (12) as a function of the matrix entry $m_{21} \equiv(1+\mu) 15 / 2 L_{B}$, with $\mu=0$ corresponding to vanishing emittance growth:

$$
\varepsilon_{x}^{2}=\varepsilon_{x 0}^{2}+\varepsilon_{x 0} \frac{25 \mu^{2}(15 \mu+13)}{48(5 \mu+3)} \theta^{2} L_{B}^{3}\left(\frac{d \sigma_{\delta}}{d s}\right)^{2}
$$

In the above expression

$$
\frac{d \sigma_{\delta}}{d s} \simeq 0.246 \times N_{b} \frac{r_{c} \theta^{2 / 3}}{\gamma \sigma_{z}^{4 / 3} L_{B}^{2 / 3}},
$$

is the rms energy spread per unit arc length induced by CSR in a bend on a Gaussian bunch $[5,15]$. We employed the macroparticle code ELEGANT [16] to simulate transport through a three-bend isochronous achromat invoking the same steady-state free-space 1D model of CSR adopted here. See Fig. 2 for the horizontal dispersion and beta function and Fig. 3 for a plot of $R_{16}^{s \rightarrow s_{f}}$ and $R_{26}^{s \rightarrow s_{f}}$.

For simplicity, we built the beam lines for each of the two sections between the dipoles using only 2 (focusing) quadrupoles. (In doing so we lose control of the matching in the vertical plane. Vertical motion, however, is irrelevant here and in the simulations we set the vertical emittance to zero).

The result from the ELEGANT simulations (red dots) and the expected emittance growth based on Eq. (32), red line, are shown in Fig. 4. As predicted by the model, essentially no emitttance growth is observed for $\mu=0$. We should note that the various data points in the figure correspond to

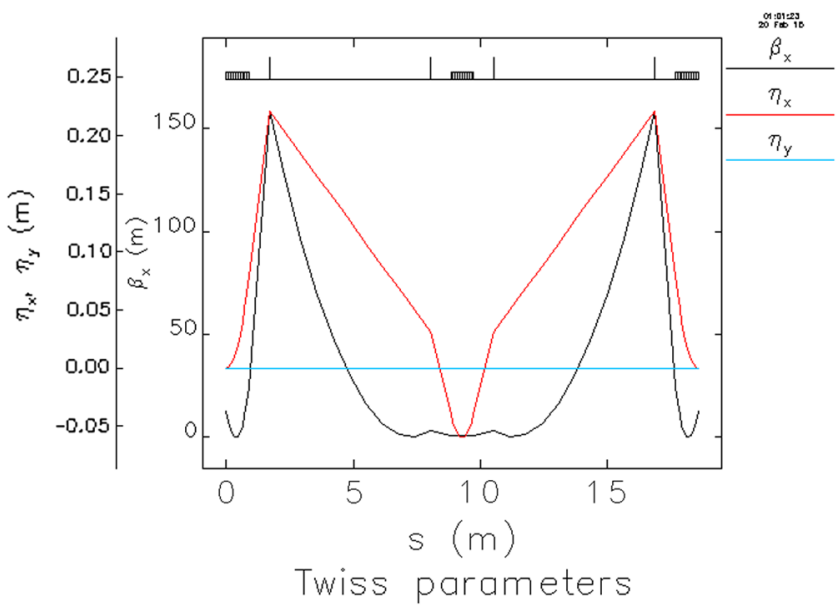

FIG. 2. Beam line layout and Twiss functions for the optimum value $m_{21}=15 / 2 L_{B}$. ELEGANT simulations. 

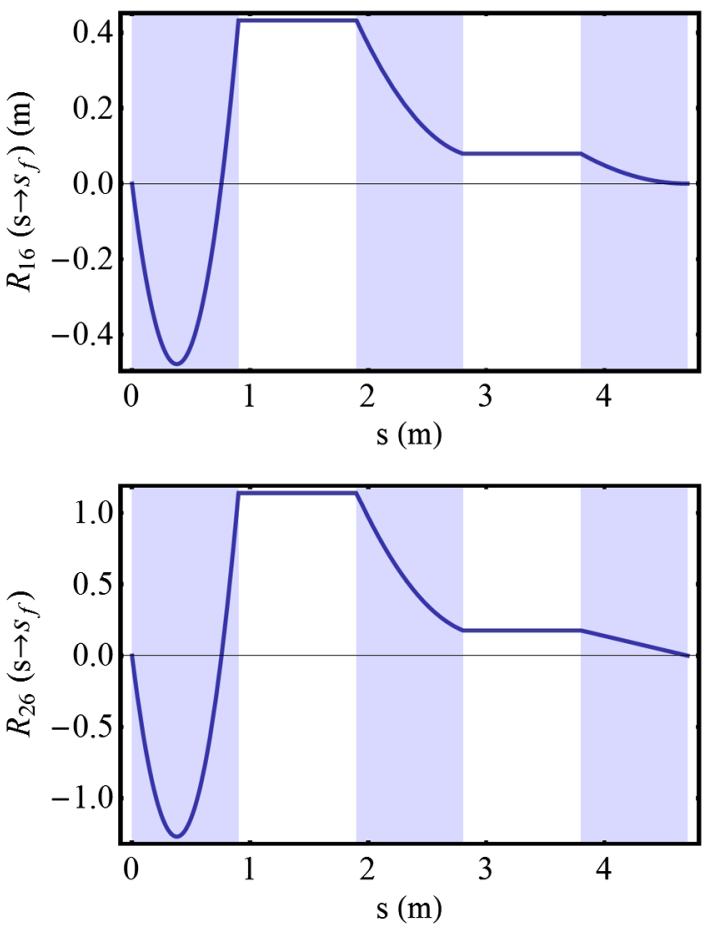

FIG. 3. Functions $R_{16}^{s \rightarrow s_{f}}$ and $R_{26}^{s \rightarrow s_{f}}$ for the optimum value $m_{21}=15 / 2 L_{B}$. The shaded areas highlight the dipoles. For better illustration, we shortened the length of the sections between the dipoles (where these functions are constant) to $1 \mathrm{~m}$ compared to Fig. 3. The sum of the integrals of the two curves within the shaded area vanish [see Eq. (14) and (15)] resulting into no projected emittance growth from CSR.

slightly different beam line lengths and different quadrupole settings, as needed to enforce the desired value of $m_{21}$, while the dipole lengths and bend angles are kept fixed.

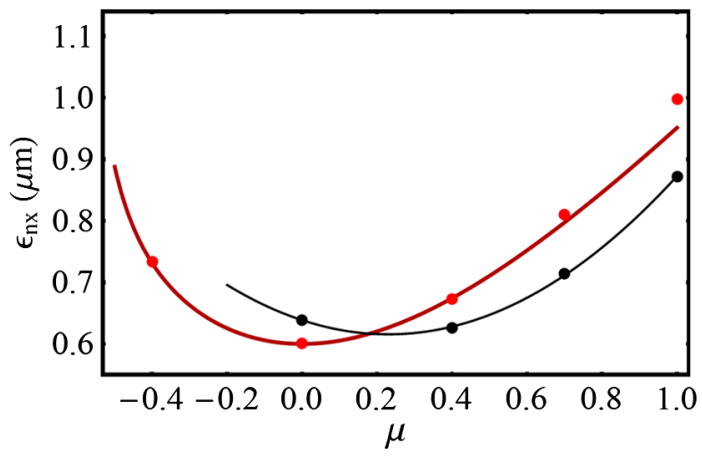

FIG. 4. Normalized projected rms emittance observed at the exit of the achromat as predicted by Eq. (32), red line, and determined by ELEGANT simulations (red dots) $v s$. $\mu$, parametrizing the entry $m_{12}=15(1+\mu) L_{B} / 2$ of the transfer matrix $M$ from the exit of the first to the entrance of the second bend, see Fig. 1. The black dots are from ELEGANT simulations carried out with the more accurate model of CSR including transient effects through entrance and exit of the dipoles. The black line is a quadratic fit, helping the eye to locate the minimum.
For comparison, we report the simulation results obtained using a more accurate 1D model of CSR accounting for transient effects (black dots) [17]; CSR was included for $4 \mathrm{~m}$ in the drift downstream of the dipoles. We observe that the main effect of the transients is to shift the minimum of the emittance growth slightly, with the minimum changing only modestly.

\section{CONCLUSIONS}

The main result of this paper is a demonstration of the existence of a triple-bend isochronous achromat design that displays virtually no projected emittance growth under the assumption of a 1D stead-state free-space model of CSR. The existence of this design setting is somewhat surprising as the problem is overconstrained; nonetheless, tuning of one independent variable turns out to suffice to satisfy both Eq. (14) and (15).

In a sense, this is the CSR equivalent of the theoretically minimum emittance (TME) lattice [13] minimizing emittance growth from incoherent synchrotron radiation.

We caution that the special design we discussed comes with somewhat extreme conditions on the lattice functions $\left[\alpha_{x}\left(s_{i}\right)=-\alpha_{x}\left(s_{f}\right) \simeq 35\right.$ ], which may be difficult to accommodate in practice, but we should add that use of unequal dipoles in the triple-bend achromat, see Eq. (24) would alleviate this difficulty.

We hope that our results may be helpful as a starting point in the search for lattice optimum designs and provide guidance on gauging the trade-off between tolerable emittance growth and desired settings of the lattice functions.

\section{ACKNOWLEDGMENTS}

Work supported by Department of Energy Contract No. DE-AC02-05CH11231. We thank P. Emma for useful discussions and review of the manuscript.

\section{APPENDIX A: ALTERNATE METHOD TO MINIMIZE CSR-INDUCED EMITTANCE GROWTH}

Suppose that at least one between $\left\langle\Delta x^{\prime 2}\right\rangle$ and $\left\langle\Delta x^{2}\right\rangle$ is nonvanishing, say $\left\langle\Delta x^{2}\right\rangle \neq 0$. If we choose (see sketch in Fig. 5)

$$
\alpha_{x}=-\beta_{x}\left\langle\Delta x^{\prime 2}\right\rangle^{1 / 2} /\left\langle\Delta x^{2}\right\rangle^{1 / 2}
$$

we can write the second term in the right-hand side of (12) representing the CSR-induced emittance growth as

$$
\begin{aligned}
& \varepsilon_{x 0}\left[\beta_{x}\left\langle\Delta x^{\prime 2}\right\rangle+2 \alpha_{x}\left\langle\Delta x^{\prime 2}\right\rangle^{1 / 2}\left\langle\Delta x^{2}\right\rangle^{1 / 2}+\gamma_{x}\left\langle\Delta x^{2}\right\rangle\right] \\
& \quad=\varepsilon_{x 0}\left\langle\Delta x^{2}\right\rangle / \beta_{x} .
\end{aligned}
$$




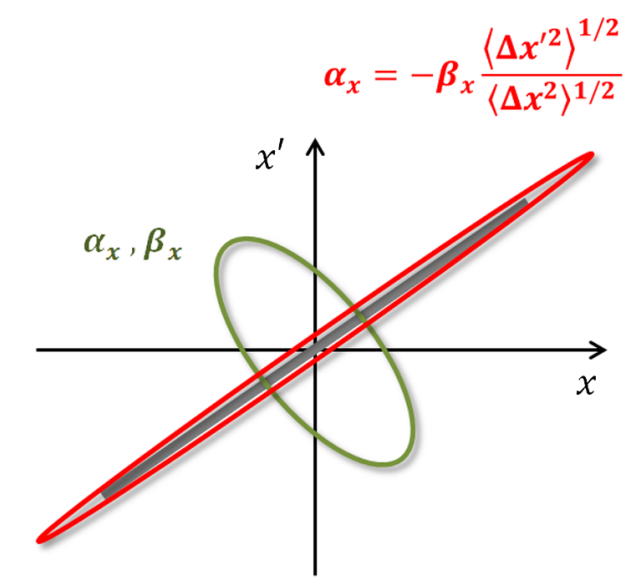

FIG. 5. The gray thick segment along the diagonal is the set of points representing the centroids of the beam slices as observed at the exit of the achromat. One can minimize the projected emittance by choosing the design Twiss functions according to Eq. (A1) to make the unperturbed beam ellipse at the exit of the beam line (red curve) align with this set. In contrast, an unperturbed beam represented by the green ellipse would maximize the projected emittance.

Again, we remind the reader that $\alpha_{x}, \beta_{x}$ and $\gamma_{x}$ are the lattice functions at the exit of the achromat. The above equation predicts vanishing emittance growth in the limit $\beta_{x} \rightarrow \infty$. Depending on the specifics of the lattice this may be a practical way to minimize the emittance growth, but large values of the beta functions for a number reasons are often not desirable.

Observe, though, that unlike the method of Sec. III the quantities $\left\langle\Delta x^{\prime 2}\right\rangle$ and $\left\langle\Delta x^{2}\right\rangle$ representing the rms spread of the beam slices spacial and angular centroids remain finite, while for certain applications (e.g., FELs) it is important that the centroids of individual beam slices stay close to the axis in order to preserve good overall beam matching and therefore favoring $\left\langle\Delta x^{\prime 2}\right\rangle=\left\langle\Delta x^{2}\right\rangle=0$. Setting aside the drawback posed by an outsized beta function [and possibly the difficulty to set $\alpha_{x}$ appropriately, Eq. (A1)], could we then argue that this emittance minimization method is less preferable than the one discussed in Sec. III? Not necessarily, because as shown below, a large $\beta_{x}$ at the exit of the achromat will have the effect of reducing the spreads $\left\langle\Delta x^{\prime 2}\right\rangle$ and $\left\langle\Delta x^{2}\right\rangle$ as observed at the end of a transport line further downstream.

Consider the most general transfer matrix for transport in the horizontal plane from the exit of the achromat to, e.g., the entrance of an FEL undulator, parametrized in terms of Twiss functions at the exit of the achromat $\left(\alpha_{x}, \beta_{x}\right)$ and entrance of the FEL $\left(\alpha_{x}^{F}, \beta_{x}^{F}\right)$ :

$$
\left(\begin{array}{cc}
\sqrt{\frac{\beta_{x}^{F}}{\beta_{x}}}\left(\cos \psi+\alpha_{x} \sin \psi\right) & \sqrt{\beta_{x}^{F} \beta_{x}} \sin \psi \\
-\frac{1+\alpha_{x} x_{x}^{F}}{\sqrt{\beta_{x}^{F} \beta_{x}}} \sin \psi+\frac{\alpha_{x}-\alpha_{x}^{F}}{\sqrt{\beta_{x}^{F} \beta_{x}}} \cos \psi & \sqrt{\frac{\beta_{x}}{\beta_{x}^{F}}}\left(\cos \psi-\alpha_{x}^{F} \sin \psi\right)
\end{array}\right) .
$$

We use this matrix to propagate the rms spread of the slice centroids to the FEL:

$$
\begin{gathered}
\left\langle\Delta x_{F}^{2}\right\rangle=\left\langle\Delta x^{2}\right\rangle \frac{\beta_{x}^{F}}{\beta_{x}} \cos ^{2} \psi, \\
\left\langle\Delta x_{F}^{\prime 2}\right\rangle=\left\langle\Delta x^{2}\right\rangle \frac{\left(\sin \psi+\alpha_{x}^{F} \cos \psi\right)^{2}}{\beta_{x} \beta_{x}^{F}} .
\end{gathered}
$$

Indeed, these two expressions show that for finite $\alpha_{x}^{F}$ and $\beta_{x}^{F}$, taking the limit $\beta_{x} \rightarrow \infty$ has the effect of reducing to zero the spreads $\left\langle\Delta x_{F}^{2}\right\rangle$ and $\left\langle\Delta x_{F}^{\prime 2}\right\rangle$ of the slices centroids as observed at the FEL. Another way to state this is to say that one can always find a symplectic transfer matrix that maps all points in the grey segment in Fig. 5 into a small region arbitrarily close to the origin of the phase space. No area-preservation theorem is violated since the segment has zero area.

\section{APPENDIX B: SELECT ENTRIES OF R-MATRIX}

For completeness we report the explicit expressions valid through 1 st order for the entries $R_{16}^{s \rightarrow s_{f}}$ and $R_{26}^{s \rightarrow s_{f}}$ for an isochronous, triple bend achromat (design with three equal dipoles).

For $s$ within the first bend:

$$
\begin{aligned}
R_{16}^{s \rightarrow s_{f}}= & L_{B} \theta_{s}^{2}\left(m_{21}^{2} L_{B}^{2}-3 m_{21} L_{B}+1\right) / 2 \theta \\
& -L_{B} \theta_{s}\left(m_{21}^{2} L_{B}^{2}-4 m_{21} L_{B}+3\right) / 2, \\
R_{26}^{s \rightarrow s_{f}}= & L_{B} \theta_{s}^{2} m_{21}\left(m_{21} L_{B}-2\right) / \theta \\
& -\theta_{s}\left(m_{21}^{2} L_{B}^{2}-3 m_{21} L_{B}+1\right),
\end{aligned}
$$

where $\theta_{s}=\left(s_{1}-s\right) / R$, and $s_{i} \leq s \leq s_{1}$. For $s$ within the second bend:

$$
\begin{aligned}
R_{16}^{s \rightarrow s_{f}}= & L_{B} \theta_{s}^{2}\left(m_{21} L_{B}-1\right) / 4 \theta \\
& +L_{B} \theta_{s}\left(m_{21} L_{B}-5\right) / 4+\theta L_{B} / 2, \\
R_{26}^{s \rightarrow s_{f}}= & m_{21} L_{B} \theta_{s}^{2} / 2 \theta+\theta_{s}\left(m_{21} L_{B}-4\right) / 2+\theta,
\end{aligned}
$$

where $\theta_{s}=\left(s_{3}-s\right) / R$, and $s_{2} \leq s \leq s_{3}$. Finally, for $s$ within the third bend: $R_{16}^{s \rightarrow s_{f}}=L_{B} \theta_{s}^{2} / 2 \theta$, and $R_{26}^{s \rightarrow s_{f}}=\theta_{s}$, where $\theta_{s}=\left(s_{f}-s\right) / R$, and $s_{4} \leq s \leq s_{f}$. For $s$ in between the bend magnets the entries $R_{16}^{s \rightarrow s_{f}}$ and $R_{26}^{s \rightarrow s_{f}}$ are constant (see Fig. 4). 
[1] E. Saldin, E. Schneidmiller, and M. Yurkov, On the coherent radiation of an electron bunch moving in an arc of a circle, Nucl. Instrum. Methods Phys. Res., Sect. A 398, 373 (1997).

[2] P. Emma and R. Brinkman, in Proceedings of the Particle Accelerator Conference, Vancouver, BC, Canada, 1997 (IEEE, New York, 1997).

[3] R. Hajima, A first-order matrix approach to the analysis of electron beam emittance growth caused by coherent synchrotron radiation, Jpn. J. Appl. Phys. 42, L974 (2003).

[4] R. Hajima, Emittance compensation in a return arc of an energy-recovery linac, Nucl. Instrum. Methods Phys. Res., Sect. A 528, 335 (2004).

[5] Y. Jiao, X. Cui, X. Huang, and G. Xu, Generic conditions for suppressing the coherent synchrotron radiation induced emittance growth in a two-dipole achromat., Phys. Rev. ST Accel. Beams 17, 060701 (2014).

[6] D. Douglas, Thomas Jefferson National Accelerator Facility Report No. JLAB-TN-98-012, 1998.

[7] D. Douglas et al., Control of Coherent Synchrotron Radiation and Micro-Bunching Effects During Transport of High Brightness Electron Beams, http://arxiv.org/abs/ 1403.2318 (2014).

[8] S. Di Mitri, M. Cornacchia, and S. Spampinati, Cancellation of Coherent Synchrotron Radiation Kicks with Optics Balance, Phys. Rev. Lett. 110, 014801 (2013).
[9] S. Di Mitri and M. Cornacchia, Transverse emittancepreserving arc compressor for high-brightness electron beam-based light sources and colliders, Europhys. Lett. 109, 62002 (2015).

[10] Y. Jing, Y. Hao, and V. N. Litvinenko, Compensating effect of the coherent synchrotron radiation in bunch compressors, Phys. Rev. ST Accel. Beams 16, 060704 (2013).

[11] C. Mitchell, J. Qiang, and P. Emma, Longitudinal pulse shaping for the suppression of coherent synchrotron radiation-induced emittance growth, Phys. Rev. ST Accel. Beams 16, 060703 (2013).

[12] M. Venturini, CSR-induced emittance growth in achromats: Linear formalism revisited, Nucl. Instrum. Methods Phys. Res., Sect. A 794, 109 (2015).

[13] S. Y. Lee, Accelerator Physics (World Scientific, Singapore, 1999).

[14] J. Corlett et al., Design concepts for a next generation light source, in Proceedings of the 35th International FreeElectron Laser Conference, New York, NY, 2013 (Brookhaven National Laboratory, Upton, NY, 2013), p. 193.

[15] Y.S. Derbenev, J. Rossbach, E. L. Saldin, and V.D. Shiltsev, Report No. TESLA-FEL 95-05.

[16] M. Borland, Report No. APS LS-287, 2000.

[17] M. Borland, Simple method for particle tracking with coherent synchrotron radiation, Phys. Rev. ST Accel. Beams 4, 070701 (2001). 